\title{
Small Bowel Mucosal Involvement and Mesenteric Mass Formation in a Young Female with Type 3 Gaucher Disease. A Case Report
}

\author{
Anthony J. Emanuel ${ }^{1}$, Nathan Holman ${ }^{2}$, Susan E. Presnell ${ }^{1}$, Cynthia T. Welsh ${ }^{1}$, Shashidhar Pai ${ }^{3}$, Shaoli Sun ${ }^{1}$
}

1) Department of Pathology

and Laboratory Medicine,

2) Division of

Gastroenterology and

Hepatology, Department of

Public Health Science,

3) Division of Medical

Genetics, Department of

Pediatrics, Medical University

of South Carolina,

Charleston, SC., USA
Address for correspondence: Shaoli Sun

Medical University of South

Carolina

171 Ashley Avenue,

Charleston, SC 29425

USA

sunsh@musc.edu
Received: 31.07 .2018

Accepted: 23.10.2018

\begin{abstract}
Gaucher Disease arises due to a deficiency in the enzyme glucocerebrosidase and is the most common lysosomal storage disease. This enzyme deficiency leads to the accumulation of glucocerebroside within macrophages (Gaucher cells) and the resulting infiltration of these cells into organs can cause clinical symptoms. There are three types of Gaucher Disease that differ based on the clinical course and the presence or absence of neurological involvement, but classically, Gaucher cell infiltrates impact a patient's spleen, liver, bone marrow and cortex. In this report, we present a case of Type 3 Gaucher Disease involving small bowel mucosa with a mesenteric mass formation. These unusual sites of Gaucher cell deposition likely led directly to uncommonly seen clinical symptoms, including small bowel obstruction and lower gastrointestinal hemorrhage.
\end{abstract}

Key words: Gaucher Disease - mesenteric mass - small bowel obstruction, gastrointestinal pathology.

Abreviations: CT: computer tomography; GC: Gaucher cells; GD: Gaucher Disease; GI: gastrointestinal; PEG: percutaneous endoscopic gastrostomy; SMV: superior mesenteric vein.

\section{INTRODUCTION}

Gaucher Disease (GD), the most common lysosomal storage disease, is characterized by a deficiency of the enzyme glucocerebrosidase. The enzyme substrate, glucocerebroside, is a cell membrane component, which is normally broken down within lysosomes of macrophages into glucose and various lipid components [1]. Accumulation of glucocerebroside within macrophages occurs as a result of the enzyme deficiency and ultimately leads to the formation of Gaucher cells (GC). Infiltration of GC within organs, where levels of glucocerebroside can approach 100 times normal values, causes clinically significant symptoms $[1,2]$. Based on the patient's clinical symptoms and disease course, GD has been divided into three types. Here, we report a patient with Type $3 \mathrm{GD}$ who presented with small bowel obstruction and an associated mesenteric mass, an unusual combination of two, previously described but rare clinical features.

\section{CASE REPORT}

A 24-year-old female with Type 3 Gaucher Disease, initially diagnosed at the age of one year due to developmental delay and seizure activity and following extensive workup at an outside hospital (records not available for review; however, in-house geneticists had been following for three years and concurred with the diagnosis), was hospitalized for bilateral pneumonia in February 2017. Her disease had been primarily managed with Imiglucerase at a dose of 4,000 units every two weeks, weekly albumin infusions, and lacosamide and levetiracetam for seizure control; other than brief lapses due to insurance issues, such as when she moved to the area in 2014, she was reportedly compliant with this therapy. At the time of hospitalization, she complained of numerous gastrointestinal (GI) symptoms, and a computed tomography (CT) scan of the abdomen showed hepatosplenomegaly and an abdominal mass (seen as early as 2014 at outside hospitals, per records). Radiological interpretation reported a $3.3 \mathrm{~cm}$ partially calcified mesenteric mass tethering of the surrounding soft tissue, small bowel wall thickening, colonic distension, and dilated mesenteric blood vessels. Colonoscopy documented a relatively patent colon with diffusely boggy mucosa. Multiple engorged vessels, likely 
representing varices, were noted in the transverse colon. The patient was not considered a surgical candidate, and she was stabilized and discharged.

The patient later presented in June 2017 with hematochezia, tachycardia, and hypotension; her hemoglobin was found to be $7.9 \mathrm{~g} / \mathrm{dL}$. She was transferred to a tertiary care center, where she stabilized after a red blood cell transfusion. A CT angiogram of her abdomen demonstrated compression of the superior mesenteric vein (SMV) with extensive varicosities; this was thought to be secondary to the previously documented mesenteric mass. Further management, such as SMV stent placement, was considered but ultimately decided against due to unclear benefit, and she was discharged after stabilization.

The patient presented again in November 2017 with abdominal pain, intractable vomiting, and poor oral intake. Small bowel obstruction was confirmed via imaging, and she was admitted for further management. Abdominal radiographs documented diffuse distension of the small bowel and colon, but no free air was detected within the peritoneum. A subsequent CT scan showed diffuse mucosal thickening of small bowel loops. Though the imaging obtained during this hospitalization focused on her gastrointestinal disease, radiology reports noted diffuse osteopenia with foci of cortical irregularity. No pathologic fractures or other acute osseous abnormalities were reported. Due to persistent electrolyte abnormalities and acute respiratory failure, she was transferred to a tertiary care facility. Notable laboratory values upon transfer included: hemoglobin $8.7 \mathrm{~g} / \mathrm{dL}, \mathrm{MCV} 68 \mathrm{fL}$, calcium 5 $\mathrm{mg} / \mathrm{dL}$, AST $256 \mathrm{U} / \mathrm{L}$, ALT $150 \mathrm{U} / \mathrm{L}$, and total protein $3.6 \mathrm{~g} / \mathrm{dL}$; all other pertinent labs were within reference ranges. The bowel obstruction did not resolve with conservative management, so a percutaneous endoscopic gastrostomy (PEG) tube was placed.

The team performing this procedure noticed prominent duodenal folds with scattered white spots, and biopsies of this area demonstrated benign small bowel mucosa with prominent macrophages in the lamina propria (Fig. 1). Various immunohistochemical and special stains were obtained to further characterize the biopsy (Table I, Fig. 2).

Following PEG placement and small bowel biopsy, the patient became increasingly lethargic. She was transferred to an intensive care unit and started on empiric antibiotics due to sepsis concerns, but she soon became unresponsive and developed tonic-clonic seizure activity. Her clinical situation

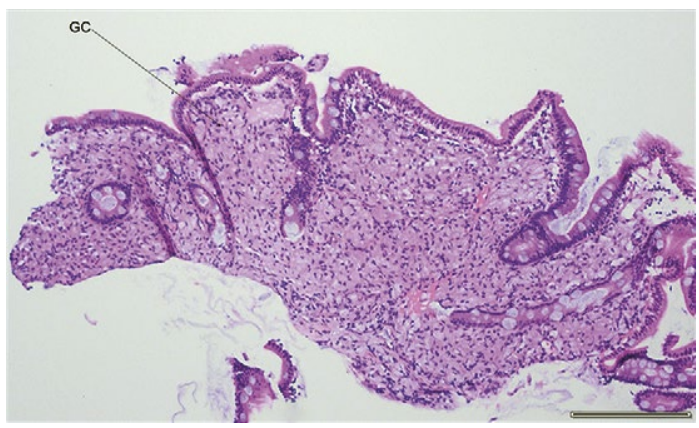

Fig. 1. Hematoxylin and eosin (H\&E, 100x) stained section of an antemortem small bowel biopsy. This biopsy shows benign small bowel with an infiltrate of foamy cells within the lamina propria; stains were requested for further characterization (Table I).
Table I. Immunohistochemical and special stains of antemortem small bowel biopsies

$\begin{array}{ll}\text { Grocott's Methenamine Silver (GMS) } & \text { Negative } \\ \text { Auramine Rhodamine } & \text { Negative } \\ \text { Tropheryma whipplei } & \text { Negative } \\ \text { S100 } & \text { Negative } \\ \text { CD68 } & \text { Positive } \\ \text { Periodic acid Schiff with diastase digestion (D-PAS) } & \text { Positive }\end{array}$

Negative staining for GMS and Auramine Rhodamine ruled against fungal and mycobacterial organisms, respectively, and Tropheryma whipplei and S100 stains were also negative. A positive CD68 stain confirmed the presence of macrophages, and a positive D-PAS (Periodic acid Schiff with diastase digestion) stain confirmed that the material within the macrophages was not entirely composed of glycogen; based on clinical history, these likely represented aggregates of Gaucher cells.

continued to deteriorate with severe multi-organ system failure, and after discussion with the patient's family, comfort care measures were instituted. She passed away, and an autopsy was requested. Due to the family's uncertainty about obtaining an autopsy as well as a language barrier, the autopsy was not performed until three weeks after her death.

Autopsy documented a thin adult female with decomposition changes. Internal examination revealed bilateral pleural effusions ( $1150 \mathrm{~mL}$ of serous fluid, combined), peritoneal ascites (350 $\mathrm{mL}$ of serous fluid), small bowel and colonic dilation, focal duodenal mucosal thickening/ hemorrhage, and a $2.5 \mathrm{~cm}$ mesenteric mass with associated fibrosis and adhesion formation (Fig. 3A). Microscopically, the tissues exhibited significant autolysis and varying amounts of microbial overgrowth, which impeded histopathological analysis of the sampled small bowel. Histology of the mesenteric mass was also impacted, but discernible features included fibrosis, nerve bundles, smooth muscle calcifications, and an aggregate of amorphous debris with macrophages. The positive staining pattern of CD68 and D-PAS on this mass suggested the presence of GC (Fig. 3B-D). Microscopic sections of the brain, which showed a relatively preserved histology, confirmed chronic brain damage by demonstrating prominent vacuolization changes, increased astrocytic activity, and numerous corpora amylacea deposits. Immunohistochemical and special stains confirmed the presence of GC that were particularly prominent in a perivascular pattern, which is commonly seen in neuropathic GD (Fig. 4).

Taken together, the microscopic findings of this case confirmed multi-organ system involvement by GC, including the mesentery and bowel. The resulting abdominal mass formation and small bowel obstruction contributed to overwhelming sepsis and multi-organ system failure. The cause of death was documented as complications of Gaucher Disease.

\section{DISCUSSION}

Gaucher Disease is an autosomal recessive disorder that belongs to a larger group of disorders known as lysosomal storage diseases [1]. As first discovered in 1966 by Brady et al., the defining characteristic of GD is a deficiency of glucocerebrosidase, an enzyme responsible for breaking down the cell membrane component glucocerebroside into 

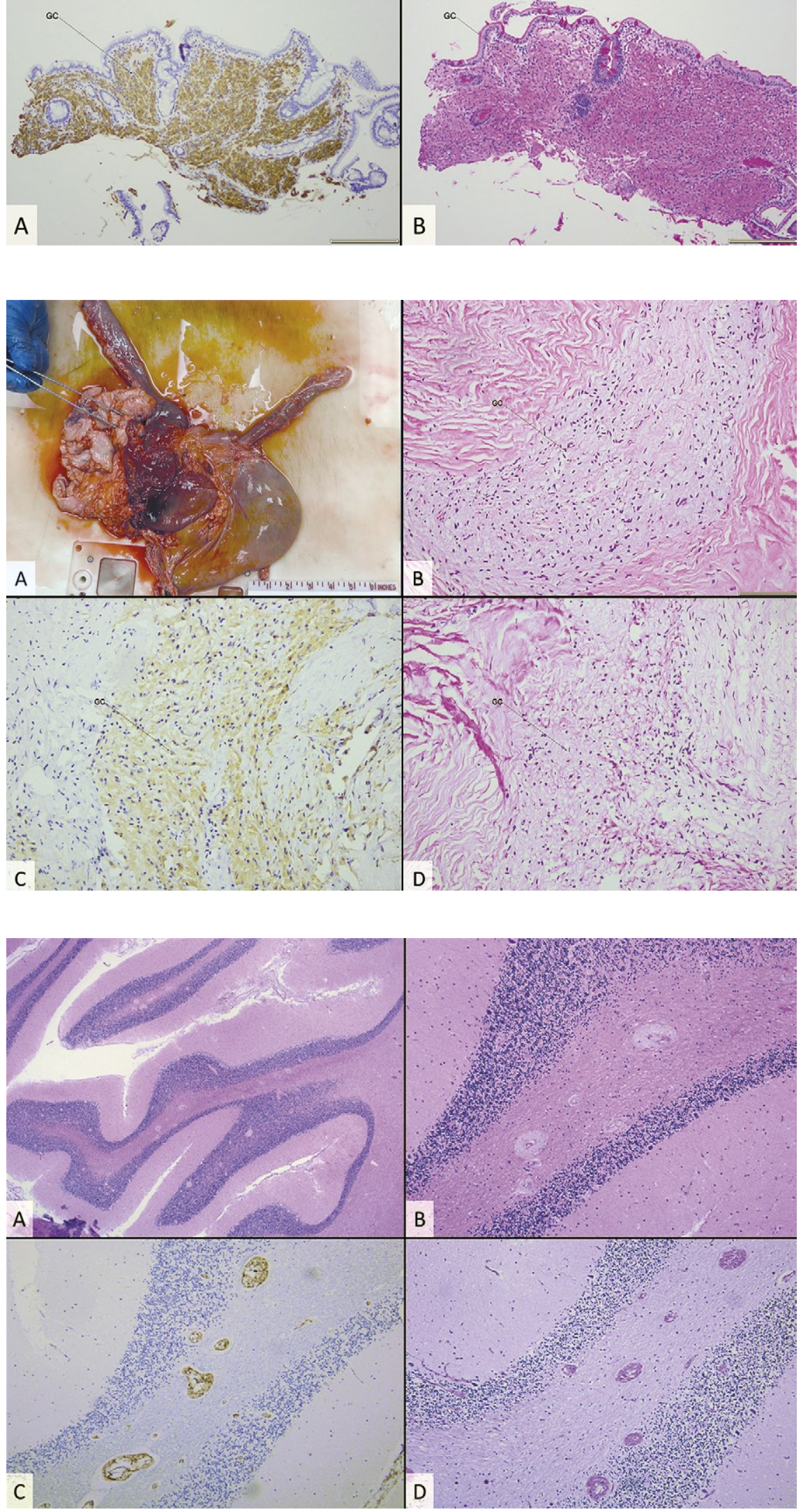

Fig. 2. A) CD68 immunohistochemical stain (100x) performed on the antemortem small bowel biopsy shows diffusely positive staining in the infiltrating cells. B) Likewise, these cells are strongly positive with a D-PAS stain $(100 \mathrm{x})$. Taken together, these findings confirm the presence of Gaucher cells within the small bowel.
Fig. 3. A) Location of the palpable mesenteric mass that was grossly identified and sampled during the autopsy (forceps). For orientation, the esophagus is to the right, and the duodenum is to the left. The mass measured $2.5 \times 1.5 \times 1 \mathrm{~cm}$, and there was associated fibrosis and involvement of the duodenal and jejunal walls. B) An $\mathrm{H} \& \mathrm{E}$ stained section $(200 \mathrm{x})$ of the mesenteric mass shows predominantly fibrosis with some areas which contain a foamy cell infiltrate. C) CD68 (200x) and D) D-PAS (200x) staining is attenuated due to tissue autolysis, but there is mild to moderate positive staining that is suggestive of Gaucher cells.
Fig. 4. A) This low power view (20x) of cerebellum demonstrates aggregates of macrophages predominantly based in the white matter tracts. B) A higher power view $(100 x)$ of cerebellum confirms the perivascular distribution of macrophages. C) Positive CD68 (100x ) and D) D-PAS (100x) staining again confirm that these macrophages represent Gaucher cells. Similar aggregates were confirmed histologically within the basal ganglia and hippocampus. glucose and ceramide [3]. The impacted gene is GBA1 on chromosome 1q21. Greater than $80 \%$ of the defects result from single nucleotide substitutions; although three specific changes account for approximately $78 \%$ of the mutations in Western countries, over 300 unique mutations have been identified $[2,4]$.
This disease can cause a wide range of symptoms based on the involved organs, and clinically, GD is subdivided into three categories. Type $1 \mathrm{GD}$, by far the most common, is usually non- neuropathic and often has a milder course with a slightly shortened lifespan. Commonly seen features include hepatosplenomegaly, thrombocytopenia, skeletal pain, and 
growth impairment. However, this varies, and differences between patients possibly reflect different genetic profiles. Type 2 GD, also known as acute neuropathic disease, is the most severe variant. It causes very early onset of symptoms and death within the first two to three years of life from rapidly progressive and overwhelming neurologic abnormalities. In addition to the findings seen in type 1 disease, type 2 commonly includes developmental delay, muscular problems such as hypertonia or rigidity, and seizures. Type $3 \mathrm{GD}$, also known as chronic neuropathic disease, is a milder but chronically progressive variant that includes many of the same findings seen in types 1 and 2 [2]. Regardless of the specific subcategory, all forms of GD tend to demonstrate visceral organ and skeletal manifestations, and there can be considerable overlap in presentation.

Body sites classically impacted by macrophage deposition in GD include the spleen, liver, bone cortex and marrow, and, in types 2 and 3 , the central nervous system $[1,2]$. However, other anatomic sites may be involved, causing patients to present with less common symptoms. We report a patient with biopsyconfirmed GC infiltration of small bowel, a complication with few cases noted following a focused literature review. In 2015, Rizk et al. [5] reported the case of an 8-year-old boy with biopsy-proven small bowel mucosal involvement by GC, and more recently in 2017, Kim et al. [6] described a set of identical twins who also exhibited gastroduodenal mucosal involvement. The present case contributes to this body of literature by demonstrating uncommonly seen small bowel disease in a patient with a long-standing history of type $3 \mathrm{GD}$.

Our patient additionally exhibited mesenteric mass formation, which had been present for at least 3 years. Based on a literature review, this is also a rarely described complication, with a case report published in 2002 by Lim et al. This group presented the case of a 13-month-old girl who was found to have a collection of GC-containing lymph nodes forming a mass in the small bowel mesentery [7]. The mesenteric mass of our patient demonstrated some CD68 and D-PAS positivity, which was consistent with a GC infiltrate. Moreover, our case also demonstrates a rare complication from such a mass in the form of lower GI hemorrhage. While there are some case reports of GI bleeding caused by various etiologies stemming from underlying GD, such as esophageal varices and gastric ulceration, to our knowledge there is no report of lower GI bleed presumed to be secondary to colonic varices as described here [8-10].

A limitation of this case report lies in the fact that the patient's original diagnosis was made at an outside institution. Despite attempts to retrieve primary documents confirming the diagnosis (namely, genotype and enzyme studies), this information was not formally reviewed by the authors or other clinicians at our institution, thereby precluding absolute confidence in the diagnosis and any potential correlation between this patient's presentation and a specific genotype. Upon the patient's initial presentation, in-house genetics discussed the case at length with the patient's prior treating physicians, and the diagnosis of GD was never questioned.

The presentation of this and similar cases demonstrates the importance of recognizing manifestations of GD beyond the well-characterized and classic hepatosplenomegaly. Our patient presented with symptoms of small bowel obstruction, and this was ultimately confirmed by imaging. It is likely that the small bowel thickening, caused by mucosal macrophage infiltration, and/or the mesenteric mass precipitated the bowel obstruction - a potential complication postulated by Lim et al. [7]. The bowel obstruction and resulting lower GI hemorrhage seen in this case are complications that are more likely to be seen in long-standing, moderate-severe cases. With enzyme replacement therapy leading to improvements in quantity and quality of life in patients with GD, and current research efforts being directed at the underlying genetic abnormalities, it is possible that involvement of unusual locations and such complications become increasingly common [1]. Treating clinicians should be cognizant of unexpected complications and quick to consider how they may relate to the underlying disease process.

\section{CONCLUSION}

This case highlights the unusual sites of Gaucher cell deposition likely led directly to uncommonly seen clinical symptoms, including small bowel obstruction and lower gastrointestinal hemorrhage.

Conflicts of interest: No conflicts of interest or sources of funding to declare.

Authors' contribution: A.E.: manuscript drafting; S.S.: concept and critical review of manuscript, review of gastrointestinal pathology; N.H.: treatment of patient and critical review of manuscript with focus on clinical course; S. Presnell: critical review of manuscript, review of autopsy proceedings/findings; C.W.: critical review of manuscript, review of neuropathology; S. Pai: clinical/genetic correlation, critical review of content. All authors read and approved the final version of the manuscript.

Informed consent: As part of the admission documents for our teaching hospital, informed consent allowing the use of this patient's health care information for the purposes of training or scientific study was provided by the patient's mother (guardian/next-of-kin). We are grateful for the willingness of the patient and family to contribute to the body of literature for this rare disease.

\section{REFERENCES}

1, Cabrera-Salazar MA, Barranger JA. Gaucher disease: review and perspectives on treatment. In: Barranger JA, Cabrera-Salazar MA. (eds.). Lysosomal Storage Disorders. New York, New York: Springer Science and Business Media; 2007:319-343. doi:10.1007/978-0-38770909-3

2. Rosenbloom BE, Weinreb NJ. Gaucher disease: a comprehensive review. Crit Rev Oncog 2013;18:163-175. doi:10.1615/ CritRevOncog.2013006060

3. Brady RO, Kanfer JN, Bradley RM, Shapiro D. Demonstration of a deficiency of glucocerebroside-cleaving enzyme in Gaucher's disease. J Clin Invest 1966;45:1112-1115. doi:10.1172/JCI105417 
4. Gaucher disease. National Institutes of Health: Genetics Home Reference website. Reviewed September 2014. Accessed May 5,2018. Available from: https://ghr.nlm.nih.gov/condition/gaucherdisease\#diagnosis

5. Rizk TM, Ariganjoye RO, Alsaeed GI. Gaucher disease: unusual presentation and mini- review. Neurosciences (Riyadh) 2015;20:271276.

6. Kim YM, Shin DH, Park SB, Cheon CK, Yoo HW. Case report of unexpected gastrointestinal involvement in type 1 Gaucher disease: comparison of eliglustat tartrate treatment and enzyme replacement therapy. BMC Med Genet 2017;18:55. doi: 10.1186/s12881-0170403-x
7. Lim AK, Vellodi A, McHugh K. Mesenteric mass in a young girl - an unusual sitefor gaucher's disease. Pediatr Radiol 2002;32:674-676. doi:10.1007/s00247-002-0761-0

8. Henderson JM, Gilinsky NH, Lee EY, Greenwood MF. Gaucher's disease complicated by bleeding esophageal varices and colonic infiltration by gaucher cells. Am J Gastroenterol 1991;86:346-348

9. Aderka D, Garfinkel D, Rothem A, Pinkhas J. Fatal bleeding from esophageal varices in a patient with Gaucher's disease. Am J Gastroenterol 1982;77:838-839.

10. Chiarugi M, Martino MC, Buccianti P, Goletti O. Bleeding gastric ulcer complicating splenosis in type 1 Gaucher's disease. Eur J Surg 1996;162:63-65. 